\title{
Transverse-Magnetic BPM Analysis of a Step-Index Slab Waveguide Expressed by a Sigmoid Function
}

\author{
Junji Yamauchi, Senior Member, IEEE, Naoya Shimada, Yuta Nito, and Hisamatsu Nakano, Fellow, IEEE
}

\begin{abstract}
Power conserving property of the propagating transverse-magnetic wave in a strongly guiding waveguide is demonstrated using the beam-propagation method with a transformed field. A step-index profile is expressed by a sigmoid function. A tilted, asymmetrical waveguide and a Y-branching waveguide with high index contrast are analyzed and discussed.
\end{abstract}

Index Terms-Beam-propagation method (BPM), high index contrast, optical waveguides.

\section{INTRODUCTION}

$\mathbf{F}$ OR THE analysis of a $z$-varying dielectric waveguide by the beam-propagation method (BPM), one is often confronted with the problem that the power is not conserved during the propagation process, particularly when simulating the transverse-magnetic (TM) waves. This is due to the fact that a simple Fresnel equation, similar to that in the transverse-electric (TE) waves, is used even for the TM analysis. Vassallo [1] clearly indicated the necessity of including the variation of the refractive index along the propagation direction. Hoekstra [2] introduced the transformation of the $H$-field to $H / n$, where $n$ is the refractive index. Subsequently, Poladian and Ladouceur [3] extended Hoekstra's idea to a more general case, and succeeded in analyzing TM waves in a tilted, graded-index waveguide. It should be noted, however, that the transformation technique does not work well for a step-index waveguide. The authors, therefore, numerically investigated the effects of including the variation of the refractive index in detail [4]. On the other hand, Ho and Lu [5] introduced a single scatter approximation for the wide-angle TM wave. A power-conserving fourth-order accurate BPM was also developed using a Padé operator [6].

We should note that a step-like change of the refractive index can be approximated by a sigmoid function, which allows us to express a step-index profile as a special case of a graded-index profile [7]. Since the sigmoid function is smooth and differentiable, we are encouraged to apply the transformation technique to the analysis of a waveguide with a step-index profile approximated by the sigmoid function. Calculation in this letter shows that the power is well conserved during the propagation

Manuscript received September 02, 2008; revised October 13, 2008. First published November 18, 2008; current version published January 16, 2009. This work was supported by MEXT Grant-in-Aid for Scientific Research (C) (19560355).

J. Yamauchi, Y. Nito, and H. Nakano are with the Faculty of Engineering, Hosei University, Koganei, Tokyo 184-8584, Japan (e-mail: j.yma@k.hosei.ac. jp).

N. Shimada is with the Fuji Electric Device Technology Co., Ltd., Nagano 390-0821, Japan.

Digital Object Identifier 10.1109/LPT.2008.2008728 process even for the simulation of a tilted, asymmetrical waveguide with high index contrast. As an application, a strongly guiding Y-branching waveguide is analyzed and discussed.

\section{FORMULATION}

Using the slowly varying envelope approximation (SVEA), a propagation equation in a two-dimensional waveguide for the TM wave is expressed as follows:

$$
\begin{aligned}
2 j k_{0} n_{0} \frac{\partial H}{\partial z} & =n^{2} \frac{\partial}{\partial x}\left(\frac{1}{n^{2}} \frac{\partial H}{\partial x}\right) \\
& +\left(k_{0}^{2}\left(n^{2}-n_{0}^{2}\right)-j k_{0} n_{0} n^{2} \frac{\partial}{\partial z}\left(\frac{1}{n^{2}}\right)\right) H
\end{aligned}
$$

where $H_{y}=H(x, z) \exp \left(-j k_{0} n_{0} z\right)$, in which $n_{0}$ is the reference index and $k_{0}$ is the free-space wavenumber. The power is evaluated by $\int|H|^{2} / n^{2} d x$.

On the other hand, using the transformation of $H_{y}=$ $n(x, z) F_{y}(x, z)$ together with the SVEA, Poladian and Ladouceur [3] derived

$$
\begin{aligned}
2 j k_{0} n_{0} \frac{\partial F}{\partial z} & =\frac{\partial^{2} F}{\partial x^{2}} \\
& +\left(k_{0}^{2}\left(n^{2}-n_{0}^{2}\right)-n \frac{\partial^{2} n^{-1}}{\partial x^{2}}-n \frac{\partial^{2} n^{-1}}{\partial z^{2}}\right) F
\end{aligned}
$$

The last term, $n \partial^{2} n^{-1} / \partial z^{2}$, can be omitted within the framework of the Fresnel approximation. It should be noted that (2) is equivalent to a TE propagation counterpart except for a different refractive index profile. The power is simply expressed as $\int|F|^{2} d x$.

The derivative of $n^{-1}$ will be singular if $n$ is not smooth, as in a step-index waveguide. To resolve this difficulty, we express a step-index profile by a smoothly varying function, i.e., the following sigmoid function is adopted:

$$
n(a, x)=\frac{n_{\mathrm{co}}-n_{\mathrm{cl}}}{1+\exp (\mp a(x \pm d))}+n_{\mathrm{cl}}
$$

where $a$ is the parameter that determines the steepness of the index change. $n_{\mathrm{co}}$ and $n_{\mathrm{cl}}$ are the refractive indexes of the core and the cladding, respectively, and $d$ is half the waveguide width.

By increasing the parameter $a$, we can make a more step-like profile. When the parameter $a$ is taken to be infinity, the refractive index profile becomes a complete step-index profile. Examples of some sigmoid functions with various values of $a$ are depicted in Fig. 1, in which $n_{\mathrm{co}}$ and $n_{\mathrm{cl}}$ are chosen to be 3.512 and 3.17 , respectively. We typically choose $a=500$ in the 


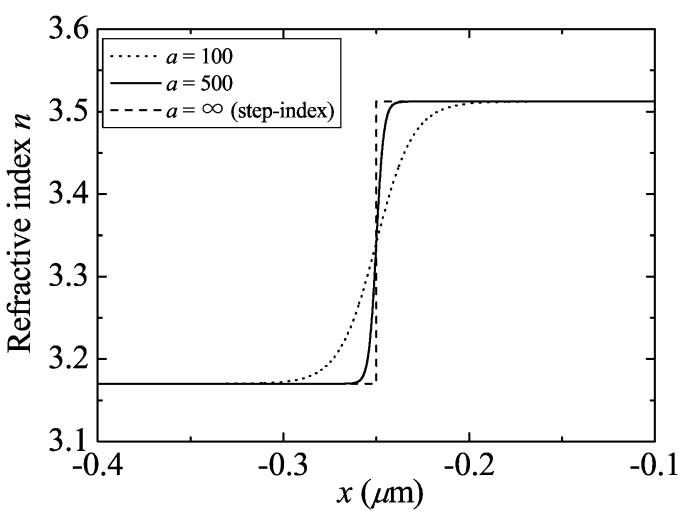

Fig. 1. Examples of sigmoid functions.

following analysis.As a result, the refractive index becomes a smooth function and differentiable, and, therefore, the conventional central difference formula with second order accuracy is employed to evaluate the derivatives in the transverse direction.

Note that the term $n \partial^{2} n^{-1} / \partial x^{2}$ can be evaluated analytically owing to the use of the sigmoid function. Preliminary eigenmode analysis, however, shows that the use of the analytical expression often results in the generation of a spurious mode depending on the choice of $\Delta x$ and $a$. As $a$ is increased, an extremely small $\Delta x$ is required to avoid the generation of the spurious mode, although the accuracy is generally better than that evaluated numerically. In this letter, we, therefore, adopt the numerical evaluation, which yields the results with reasonable accuracy, as will be seen in Section III.

\section{DISCUSSION}

A spurious loss or amplification of the propagating field of the TM wave becomes noticeable when the refractive index profile is asymmetrical with high index contrast [1]. We, therefore, deal with an asymmetrical step-index waveguide. The refractive indexes are $n_{1}=3.512, n_{2}=3.17$, and $n_{3}=1.0$, respectively. The core width is taken to be $2 d=0.5 \mu \mathrm{m}$. Before doing the propagating beam analysis, we first check the validity of using the sigmoid function in the eigenmode analysis. We calculate the fundamental mode at a wavelength of $\lambda=1.55 \mu \mathrm{m}$ using the imaginary distance procedure. The sampling widths are taken to be $\Delta x=0.005 \mu \mathrm{m}$ and $\Delta z=0.02 \mu \mathrm{m}$. Calculation shows that the propagation constant of the waveguide whose refractive index profile is approximated by the sigmoid function is $\beta=13.44079$, while the exact propagation constant of the step-index waveguide, which is obtained analytically, is $\beta=13.44297$. That is, the difference between the propagation constants is less than $0.017 \%$, which may be negligible in most waveguiding problems.

We now carry out the propagating beam analysis. The asymmetrical step-index waveguide is tilted by $\theta=5^{\circ}$, as shown in Fig. 2. The fundamental mode at a wavelength of $\lambda=1.55 \mu \mathrm{m}$ is launched. The sampling widths are taken to be the same as those used in the eigenmode analysis, except for $\Delta x=0.005 / \cos \theta \mu \mathrm{m}$. The reference index is chosen to be $n_{0}=\beta \cos \theta / k_{0}$, where $\beta$ is the propagation constant of the

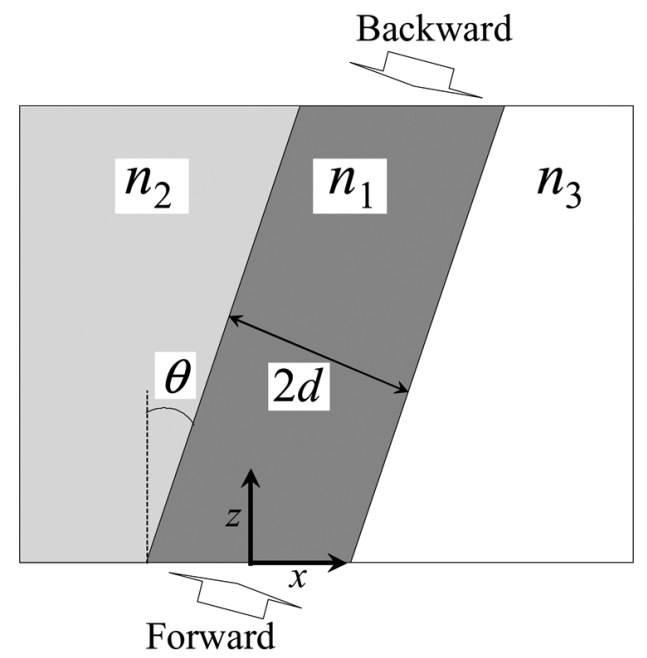

Fig. 2. Configuration of a tilted, asymmetrical waveguide.

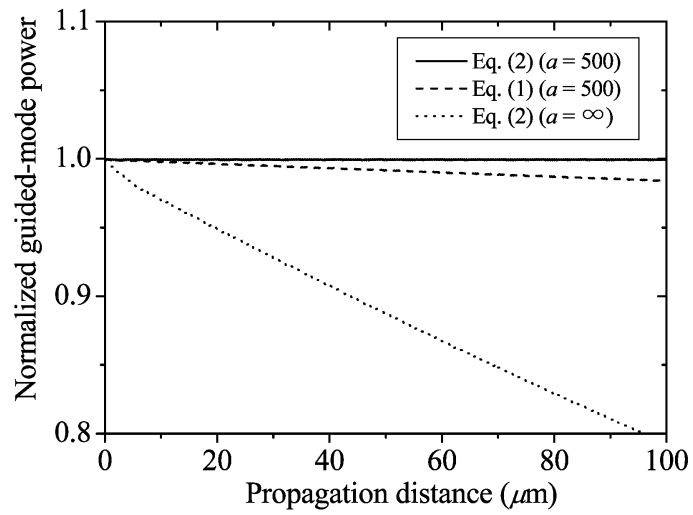

(a)

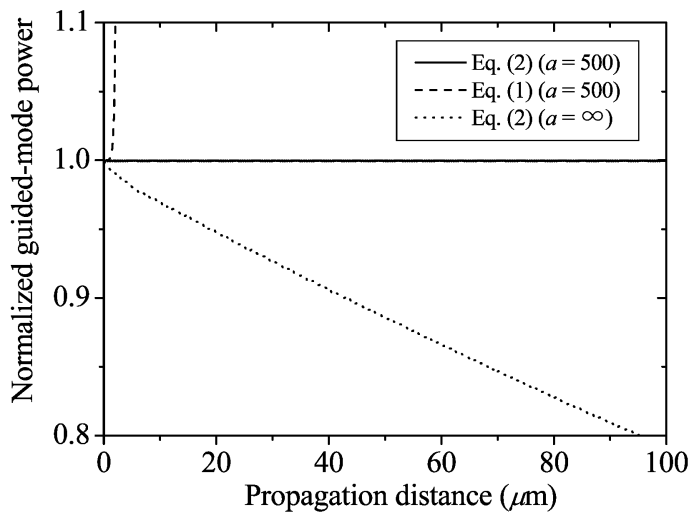

(b)

Fig. 3. Normalized guided-mode power of the TM wave as a function of propagation distance. (a) Forward. (b) Backward.

waveguide. A transparent boundary condition (TBC) [8] is imposed at the edge of the computational region. The simulation is done for both forward and backward propagation directions.

Fig. 3 shows the normalized guided-mode power as a function of propagation distance. If the numerical calculations are accurate, we do not expect any power loss (or amplification) as the eigenmode propagates, so that the normalized guided-mode power retains unity. 


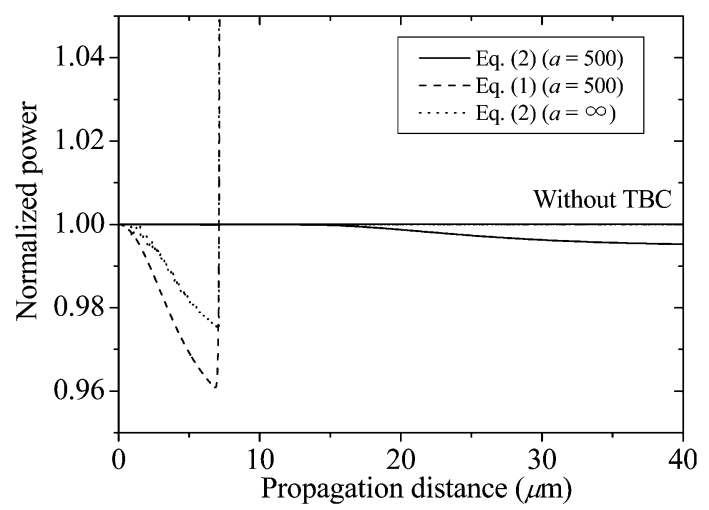

Fig. 4. Normalized power of the TM wave as a function of propagation distance in a Y-branching waveguide.

Fig. 3(a) shows the forward propagation case, and Fig. 3(b) the backward case. The solid line represents the data obtained from (2) with the sigmoid function $(a=500)$. For reference, the data obtained from (1) with the sigmoid function $(a=500)$ and that from (2) with a step-index profile $(a=\infty)$ are also shown by the broken and dotted lines, respectively. It is found that the power obtained from (2) with the sigmoid function $(a=500)$ is well conserved. In contrast, the use of (1) exhibits a spurious loss or amplification, particularly for the backward propagation case. Fig. 3 also shows that the use of (2) with a step-index profile does not work well in terms of power conservation. As a result, the use of (2) together with the sigmoid function is significantly effective.

It should be noted that the above-mentioned analysis is performed using the exact propagation constant as the reference index. We also check the dependence of the power on the reference index. Calculation shows that the power variation is less than $1 \%$ over a reference index variation of $\pm 30 \%$. This property is almost the same as that observed in the TE mode analysis.

As an application, we simulate a $40-\mu \mathrm{m}$-long Y-branching waveguide, the centers of which describe the two curves $x=$ $\pm[1-\cos (\pi z / 40)]$ in the lateral direction. We intentionally treat a strongly guiding waveguide, so that the refractive indexes of the core and the cladding are chosen to be $n_{\mathrm{co}}=3.512$ and $n_{\mathrm{cl}}=1.0$, respectively. The core width of the input (output) waveguide is taken to be $2 d=0.3 \mu \mathrm{m}$. A wavelength of $\lambda=$ $1.55 \mu \mathrm{m}$ is used and the fundamental mode is excited from the input port. The reference index is fixed to be the effective index of the input waveguide.

Fig. 4 shows the normalized power as a function of propagation distance. We can again find that the use of (2) together with the sigmoid function leads to stable results. Note that the slight loss observed with the present scheme [(2) with $a=500$ ] is due to the radiation towards the absorbing boundaries. When the TBC is removed, we can confirm that the power is well conserved, as clearly indicated in Fig. 4.

We finally comment on the extension of the present technique to a wide-angle BPM. For example, the formulation of the $(1,1)$ Padé-operator-based BPM seems to be straightforward. Our calculation, however, shows that the power conservation property is degraded when the Padé operator is used, although the numerical stability is maintained. This degradation is larger than that observed in the TE case [9]. The extension to the wide-angle BPM must be the subject of future research.

\section{CONCLUSION}

We have simulated the TM wave propagation along an asymmetrical step-index waveguide with high index contrast, which is expressed using a sigmoid function. The propagating field is satisfactorily analyzed with the power being well conserved. No computational instability is observed during either the forward or the backward propagation process. The present method is also effective for analyzing a Y-branching waveguide with high index contrast. The application of the sigmoid function to a three-dimensional problem is yet to be researched.

\section{REFERENCES}

[1] C. Vassallo, "Difficulty with vectorial BPM," Electron. Lett., vol. 33 , no. 1, pp. 61-62, Jan. 1997.

[2] H. J. W. M. Hoekstra, "On beam propagation methods for modeling in integrated optics," Opt. Quantum Electron., vol. 29, no. 2, pp. 157-171, Feb. 1997.

[3] L. Poladian and F. Ladouceur, "Unification of TE and TM beam propagation algorithms," IEEE Photon. Technol. Lett., vol. 10, no. 1, pp. 105-107, Jan. 1998.

[4] J. Yamauchi, K. Matsubara, T. Tsuda, and H. Nakano, "Normconserving finite-difference beam propagation method for TM wave analysis in step-index waveguides," J. Lightw. Technol., vol. 18, no. 5, pp. 721-728, May 2000.

[5] P. L. Ho and Y. Y. Lu, "Improving the beam propagation method for TM polarization," Opt. Quantum Electron., vol. 35, no. 4, pp. 507-519, Apr. 2003.

[6] J. Yamauchi, K. Sumida, and H. Nakano, "Analysis of a polarization splitter with a multilayer filter using a Padé-operator-based power-conserving fourth-order accurate beam-propagation method," IEEE Photon. Technol. Lett., vol. 18, no. 17, pp. 1858-1860, Sep. 1, 2006.

[7] J. Yamauchi, N. Shimada, and H. Nakano, "Semivectorial BPM analysis of an asymmetrical step-index slab waveguide using a sigmoid function," in 5th Workshop on Fibers and Optical Passive Components, Taipei, Taiwan, Dec. 2007, Paper TH3A-4.

[8] G. R. Hadley, "Transparent boundary condition for beam propagation," Opt. Lett., vol. 16, no. 9, pp. 624-626, May 1991.

[9] C. Vassallo, "Wide-angle BPM and power conservation," Electron. Lett., vol. 31, no. 2, pp. 130-131, Jan. 1995. 